\title{
Plano Brasil sem Miséria: a frágil receita disponível dos municípios de Minas Gerais para a proposta de custeio da Atenção Básica
}

\section{| ${ }^{1}$ José Rivaldo Melo de França, ${ }^{2}$ Nilson do Rosário Costa |}

Resumo: O artigo analisa a sustentabilidade econômico-financeira requerida para a cobertura da Atenção Básica $(\mathrm{AB})$ nos municípios de Minas Gerais selecionados pelo Governo Federal para atuação em saúde no âmbito do Plano Brasil sem Miséria (BSM). Para desenvolver o estudo, foram pesquisadas as bases de dados do Sistema de Informações sobre Orçamentos Públicos em Saúde (Siops/MS), Sala de Apoio à Gestão Estratégica (Sage/MS) e Instituto Brasileiro de Geografia e Estatística (IBGE). Os dados pesquisados foram tratados por métodos estatísticos de forma a conduzir aos resultados. Para caracterizar o problema, foram adotados portes populacionais. Os indicadores utilizados foram criados por Pereira et al. (2006). Utilizaram-se também indicadores testados por Mendes (2010). A pesquisa foi estruturada em três dimensões: recursos originários das receitas disponíveis (RD) e das transferências do SUS; despesas municipais com saúde, respectiva alocação municipal; e transferências federais para $\mathrm{AB}$, com a cobertura das linhas de atuação estruturantes. Os resultados permitem concluir que para os municípios com até 50 mil habitantes, 95,2\% do total, ampliarem suas metas ou linhas de atuação nas propostas do BSM necessitarão aprimorar sua capacidade tributária ou recorrerem a recursos suplementares das demais esferas, dados a exígua margem de RD em que operam e os elevados níveis de cobertura em $\mathrm{AB}$ que já atingiram utilizando, principalmente, suas transferências constitucionais e legais.

> Palavras-chave: políticas públicas; saúde pública; gestão pública; financiamento do SUS; Brasil sem Miséria.

\author{
1 Doutor em Saúde Pública, \\ Escola de Nacional de Saúde \\ Pública Sérgio Arouca/ \\ Fiocruz; assessor técnico da \\ Subsecretaria de Planejamento \\ e Orçamento da Secretaria \\ Executiva do Ministério da \\ Saúde, Brasília, Brasil. Endereço \\ eletrônico: jrmf1@uol.com.br \\ 2 Doutor em Arquitetura e \\ Urbanismo pela Universidade \\ de São Paulo; professor titular \\ da Fundação Oswaldo Cruz. \\ Rio de Janeiro, Brasil. Endereço \\ eletrônico: nilson@ensp. \\ fiocruz.br
}

Recebido em: 20/08/2013. Aprovado em: 18/12/2013. 
O presente artigo analisa a sustentabilidade econômico-financeira municipal requerida para a cobertura da Atenção Básica $(\mathrm{AB})$ no âmbito do Plano Brasil sem Miséria (BSM) no estado de Minas Gerais. Esse plano foi instituído pelo Governo Federal, por meio do Decreto nº. 7.492, de 2 de junho de 2011, como finalidade "de superar a situação de extrema pobreza da população em todo o território nacional, por meio da integração e articulação de políticas, programas e açôes".

$\mathrm{O}$ conceito de sustentabilidade econômico-financeira corresponde à capacidade do município para arcar, em curto e médio prazo, com os aportes financeiros da sua competência tributária e das transferências constitucionais e legais, além das do Sistema Único de Saúde (SUS), para a cobertura das propostas do Ministério da Saúde (MS), ao estabelecer compromissos públicos para o aperfeiçoamento e a ampliação do acesso a bens e serviços de saúde na esfera do BSM em Minas Gerais.

Para alguns autores (PORTELA; RIBEIRO, 2009; CÂMARA; BERMUDEZ, 2004), a sustentabilidade não envolve apenas a dimensão econômica, ou seja, a capacidade de gerar receitas tributárias e de receber as transferências constitucionais e legais (RD), mas também o comprometimento político, na continuidade e coerência dessas intervenções, evitando, dessa forma, as dimensões ideológicas e circunstanciais. Para efeito deste trabalho, serão levadas em consideração, além da dimensão econômica, algumas variáveis condicionantes e contextuais em que se desenvolvem as ações de $\mathrm{AB}$ nos municípios do BSM.

Este artigo procura determinar um perfil dos municípios selecionados com relação às RD e às transferências do SUS, bem como o potencial de aplicação em açōes de saúde. O BSM fundamenta a atuação governamental em três eixos: garantia de renda, acesso a serviços públicos e inclusão produtiva. O setor saúde atua no eixo "acesso a serviços públicos". Fundamentalmente, essa atuação setorial está relacionada ao nível básico de atenção, uma vez que a busca ativa que potencializa o acesso às açôes propostas operacionaliza-se por meio das Equipes de Saúde da Família (ESF), mais precisamente, intermediada pelos Agentes Comunitários de Saúde (ACS). 
A atuação do MS fundamenta-se na pactuação com os demais entes federados

para o compartilhamento de responsabilidades em seis linhas de ação:

1. Atenção Básica em Saúde: construção, reforma e ampliação de Unidades Básicas de Saúde (UBS); implantação de novos ACS; implantação de consultórios na rua; vinculação das ESF ao Programa Saúde na Escola (PSE) e participação dos municípios prioritários no Programa Nacional de Melhoria do Acesso e da Qualidade da Atenção Básica (PMAQ); acompanhamento das condicionalidades do Programa Bolsa Família; adesão ao programa de atenção ao acompanhamento qualificado ao período pré-natal.

2. Saúde Bucal: implantação de consultórios, centros de especialidades odontológicas e unidades odontológicas móveis; fornecimento de próteses dentárias.

3. Saneamento Básico: fornecimento de cisternas; implantação de sistemas de abastecimento de água em comunidades quilombolas, rurais e aldeias indígenas; construção de poços artesianos; plano de capacitações para promoção e controle da qualidade da água das cisternas na região do semiárido.

4. Programa Olhar Brasil: consultas oftalmológicas e fornecimento de óculos.

5. Programa Aqui Tem Farmácia Popular: fortalecimento do Programa Farmácia Popular do Brasil, para a intensificação do fornecimento gratuito de medicamentos básicos para diabetes e hipertensão e com desconto para prescrições de casos de asma, rinite, mal de Parkinson, osteoporose, glaucoma, contraceptivos e fraldas geriátricas.

6. Controle das doenças negligenciadas: concentração de esforços para intensificação do diagnóstico e tratamento de hanseníase; tracoma e geohelmintíases; e busca do diagnóstico precoce e tratamento de portadores de Schistosoma Mansoni.

Essas ações estão previstas no Plano Plurianual 2012-2015 e têm recursos federais assegurados pela Lei Orçamentária Anual de 2013 para as metas municipais, anteriormente programadas. As metas poderão ser alteradas de acordo com modificações que virão a ocorrer nas previsões locais, em consequência das propostas do BSM. As alterações poderão ser tanto no sentido de expansão como 
1232 de redução de metas e dependem de negociações pela Comissão Intergestores Tripartite (CIT). No entanto, para o caso de expansão de metas, não há previsão de aportes de recursos federais adicionais, específicos para ações do BSM. Isso significa que os ajustes orçamentários decorrentes da expansão de metas serão de responsabilidade financeira municipal ou estadual.

Para 2012 não ocorreu expansão de metas. O MS concentrou-se apenas no monitoramento das metas previstas, o que não invalida que venha a ocorrer nos anos seguintes. A situação de execução orçamentária do MS, em 2012, acusou limitações de realização financeira dos valores orçados devido ao contingenciamento de R $\$ 5$ bilhôes determinado pelo Decreto $\mathrm{n}^{\circ}$ 7.680, de 17 de fevereiro de 2012, não recuperados até o final do exercício. A execução federal ao final do ano acusou déficit de empenho de $\mathrm{R} \$ 9,1$ bilhões em relação à dotação orçamentária atualizada. Dos valores empenhados, $\mathrm{R} \$ 8,3$ bilhões foram inscritos em restos a pagar, resultando numa defasagem de execução de $\mathrm{R}$ \$ 17,4 bilhões em relação ao previsto.

A hipótese central deste estudo é de que os municípios eleitos pelo MS para atuação do BSM em Minas Gerais podem apresentar fragilidade de receitas próprias para arcarem com recursos adicionais para as metas de atenção básica propostas pelo MS. Na literatura nacional, poucos estudos examinaram as questōes relacionadas à sustentabilidade econômico-financeira municipal para fazer frente, em curto e médio prazo, à cobertura das ações de saúde, principalmente com relação à ampliação local de metas de linhas de atuação programáticas.

No campo do financiamento, grande parte dos estudos avaliativos do SUS se volta para as fontes e a suficiência de recursos para o setor. O âmbito da sustentabilidade econômico-financeira foi examinado, sob o ponto de vista do potencial municipal, por Mendes (2010), segundo metodologia desenvolvida por Mendes (2005), com a finalidade de determinar um perfil do padrão de financiamento e gasto do SUS nos 39 municípios que compõem a região metropolitana de São Paulo, no período de 2002 a 2008. Os principais achados da pesquisa indicaram que, no conjunto de municípios, o grau de dependência em relação aos impostos arrecadados é de 54,01\%, o que significa que da receita total dos municípios, pouco mais da metade tem origem na sua estrutura 
tributária. Indicou que esses municípios necessitam aprimorar sua capacidade de coleta para ampliar a participação de impostos na receita disponível, elevando, dessa forma, sua autonomia financeira para custear as políticas públicas em geral e potencializar a capacidade de gasto em saúde, em particular. O mesmo estudo verificou que, no período analisado, a participação das transferências do SUS foi crescente, o que indica elevação do grau de dependência dos recursos federais e encolhimento da propensão municipal em custear as ações de saúde.

A propósito dos achados de Mendes (2010), estudo empírico desenvolvido por Afonso e Araújo (2000) demonstra a evolução da capacidade de arrecadação, por meio da análise da geração de receitas da competência tributária municipal e da RD. Traça um painel das perspectivas de melhoria da capacidade de arrecadação, levando em consideração o aperfeiçoamento do esforço próprio de coleta tributária.

Já Rocha et al. (2012) desenvolveram uma metodologia para avaliar o volume de gastos municipais em saúde, procurando responder à indagação persistente quando se trata do financiamento do SUS: existe insuficiência de recursos ou ineficiência no seu uso? A partir da comparação entre o dimensionamento da demanda e o gasto público consumado, pressupõe que se o gasto efetivamente realizado está abaixo do demandado, não há, portanto, necessidade de recursos adicionais. Em caso de o quantitativo ofertado possibilitar folga de recursos, será possível reduzir os aportes estimados, guardando condição de uma aplicação de recursos municipais eficiente.

Ainda nesse percurso investigativo, outra linha de estudo põe centralidade na sustentabilidade financeira dos municípios para o financiamento, em curto e médio prazo, de determinada forma de atuação setorial. Nesse sentido, Pereira et al. (2006) construíram indicadores de sustentabilidade financeira para analisar o Programa de Saúde da Família (PSF), em cidades com mais de 100 mil habitantes, nos estados do Amapá, Maranhão, Pará e Tocantins, com adesão ao Programa de Expansão e Consolidação da Saúde da Família (Proesf), cujo objetivo seria incentivar a ampliação do PSF nas grandes cidades brasileiras. A pesquisa teve como finalidade avaliar a capacidade desses municípios em arcar com os gastos, diretos e indiretamente incorridos, em médio e longo prazo, decorrentes da evolução esperada para o PSF. 
O modelo adotado, observadas suas limitaçōes, sugeriu uma forma de classificação de desempenho econômico-financeiro passível de auxiliar a capacidade da União e dos estados de "ampliar a consistência e dar maior particularidade" aos incentivos financeiros concedidos para estimular o desenvolvimento das atividades do PSF nos municípios com mais de 100 mil habitantes.

Outro estudo que investigou a sustentabilidade econômico-financeira dos municípios brasileiros, com mais de 100 mil habitantes, para fazer frente ao desenvolvimento do PSF, foi elaborado por Portela e Ribeiro (2011). Utilizando indicadores desenvolvidos por Pereira et al. (2006), os autores examinaram o esforço municipal para a cobertura financeira da $A B$, segundo as regióes geográficas, portes municipais e participação no Proesf. Concluiu que a análise por região geográfica foi a que melhor caracterizou os municípios por meio dos indicadores utilizados. Os achados da pesquisa indicaram a Região Sudeste como a de maior capacidade fiscal, maior adequação ao cumprimento da Emenda Constitucional 29 e a associação dessas variáveis a maiores médias de despesa per capita potencial em saúde.

Com relação ao porte populacional, a pesquisa constatou que, nos municípios com mais de 100 mil habitantes, também se confirma o pressuposto de que quanto menos populoso o município, mais altas as coberturas das Equipes de Saúde da Família (ESF), apesar de essas médias terem pouca significância com relação ao cumprimento da meta proposta pelo Proesf.

Os estudos examinados produziram subsídios para projeções de médio e longo prazo para o desenvolvimento localizado e territorialmente abrangente do SUS e da Estratégia Saúde da Família. A geração de indicativos importantes para a estruturação de intervenções sanitárias estimula iniciativas de aplicação das metodologias já testadas para outros tipos de avaliações. Com esse fito, os indicadores já construídos, acrescidos de outros coerentes com o objetivo pretendido nesta pesquisa, permitem avaliar se os municípios de Minas Gerais, com pactuação de metas no âmbito do BSM, têm possibilidade de fazer frente aos compromissos acordados e desenvolver ações no nível pactuado em médio e longo prazo. 


\section{Metodologia}

Foi realizado estudo de dados de painel sobre o comportamento dos municípios indicados para atuação do BSM em Minas Gerais, com relação à sustentabilidade econômico-financeira para a cobertura de açôes de $\mathrm{AB}$. Foram consideradas algumas premissas que dão conformidade ao estudo. Com relação às possiblidades de disponibilização de recursos próprios, considerouse o conceito de Receita Disponível (RD) que corresponde à arrecadação tributária de competência municipal, considerada como base vinculável para fins do estabelecido pela Emenda Constitucional 29 (EC 29), adicionada das transferências constitucionais e legais para os municípios, realizadas pelos demais entes federativos (MENDES, 2005).

Ainda, observando Mendes (2005), as despesas consideradas correspondem àquelas liquidadas originárias do orçamento municipal, não levando em consideração despesas com saúde realizadas no município, porém de execução direta dos outros entes federativos. As bases de dados pesquisadas foram: Sistema de Informações sobre Orçamentos Públicos em Saúde (Siops), cuja função é acompanhar a apuração das receitas totais e os gastos em ações e serviços públicos de saúde, de acordo com a Lei Complementar no 141, de 13 de janeiro de 2012, que regulamentou a EC 29. A captação do banco de dados diretamente do Siops ocorreu em 24 de outubro de 2012; Sala de Apoio à Gestão Estratégica (Sage) do MS, consulta realizada em 06 de dezembro de 2012. Os percentuais de cobertura foram captados diretamente da Sage, assim como as transferências federais por linhas de atuação.

A população que serve de referência para os per capitas financeiros foi extraída do Censo 2010, realizado pelo Instituto Brasileiro de Geografia e Estatística (IBGE). Os dados financeiros foram atualizados para 2011 pelo Índice Nacional de Preços ao Consumidor (INPC), calculado pelo IBGE, índice usualmente utilizado pelo governo para as atualizaçōes orçamentárias. As principais referências utilizadas foram o Demonstrativo da Lei de Responsabilidade Fiscal divulgado pelo Siops e os valores transferidos por meio dos Blocos de Financiamento do SUS, conforme divulgados pela Sage. 
As observações atípicas (outliers) percebidas ao longo da série, em algumas variáveis, não foram consideradas. Essa atipicidade, ocasionada por motivos conjunturais, consiste em valores muito elevados do recolhimento tributário de determinados municípios, alçando níveis bem além de outros de porte semelhante. Os outliers foram controlados utilizando-se a função gráfica boxplot, ${ }^{1}$ considerando-se faixas de valores correspondentes a 1,5 de distância interquartílica. (SOARES, 1999).

A amostra pesquisada corresponde aos 188 municípios de Minas Gerais definidos pelo MS para atuação do BSM em outubro de 2012. Esses municípios são caracterizados pelo alto grau de presença de famílias onde ocorrem rendimentos mensais per capita de até $\mathrm{R} \$ 70,00$. Nessa situação foram mapeadas, no Brasil, cerca 16 milhões de pessoas residentes em 2.360 municípios em 2011. Esses municípios estão concentrados, principalmente, em três das 13 Macrorregiōes de Saúde delimitadas pela Secretaria de Estado da Saúde de Minas Gerais: Nordeste (Teófilo Otoni) com 84,21\% dos seus municípios; Jequitinhonha (Diamantina) com 62,07\% dos seus municípios e Norte (Montes Claros) com 47,67\% dos seus municípios. As demais tiveram variaçôes de 0 a $17,44 \%$ dos seus municípios selecionados.

Devido à concentração espacial predominante dos municípios proporcionarem relativa homogeneidade regional em termos socioeconômicos, optou-se pela estratificação por porte populacional. Essa classificação permitirá maior amplitude de análise com relação à capacidade fiscal e de gestão municipal de acordo com o porte populacional. Para caracterizar o problema, foram adotados portes demográficos a partir da população dimensionada pelo IBGE. Com esse propósito, foram delimitadas faixas municipais com ênfase nos municípios menos populosos, onde se observa maior incidência de selecionados.

\section{Quadro 1. Estratificação dos municípios segundo o porte populacional}

\begin{tabular}{|lcc|}
\hline Porte Municipal & Faixas Populacionais & No de Municípios \\
\hline 1 & Até 10 mil habitantes & 115 \\
\hline 2 & Mais de 10 mil até 50 mil habitantes & 64 \\
\hline 3 & Mais de 50 mil até 100 mil habitantes & 3 \\
\hline 4 & Mais de 100 mil & 6 \\
\hline
\end{tabular}

FONTE: Elaboração própria, a partir da contagem populacional do Censo 2010 realizado pelo IBGE. 
Os indicadores utilizados foram criados por Pereira et al. (2006) e testados com significância por Portela e Ribeiro (2011). Utilizaram-se também outros já testados Mendes (2010), além dos de cobertura e financiamento das ações estruturantes da $\mathrm{AB}$ introduzidos pelos autores. Observaçôes preliminares nas bases de dados definiram o período de 2004 a 2011 por apresentarem grau de consistência das informações mais elevado.

A pesquisa foi estruturada em três dimensões: a) recursos originários das receitas disponíveis e das transferências do SUS; b) despesas municipais com saúde; e c) alocação municipal e transferências federais para atenção básica e evolução da cobertura das linhas de atuação estruturantes. Em cada dimensão foram calculadas as médias por portes municipais correspondentes às variáveis analisadas.

\section{Dimensão das Receitas Disponíveis (RD)}

Foram analisadas as disponibilidades de recursos municipais geradas da estrutura tributária de competência própria e sua participação na formação da RD. Essa relação indica o grau de independência que o ente federado municipal tem para o gasto em saúde. Supõe-se que quanto mais elevada for a relação, maior será a autonomia do gestor municipal para dispor de recursos para a sustentação do gasto público em saúde executado pela esfera administrativa (MENDES, 2010). Para o autor, esse indicador também revela o esforço de arrecadação de impostos de responsabilidade municipal para a cobertura das políticas públicas em geral.

A análise da evolução da arrecadação de impostos informa, principalmente, a relação existente entre o volume coletado e as variações cíclicas da economia. $\mathrm{Na}$ variável impostos foram excluídos os outliers detectados.

Com relação à situação do cumprimento da EC 29, Pereira et al. (2006) classificam como positiva para o conjunto de municípios em que todos empregaram recursos de RD acima de 15\% do gasto com ações e serviços de saúde e negativa a situação inversa. Foram analisados os portes municipais por médias e Coeficiente de Variação $(\mathrm{CV})^{2}$ para que se determine o peso dos municípios em situação positiva, obtendo-se dessa forma um perfil do cumprimento da EC 29 no conjunto de municípios.

Os indicadores de sustentabilidade econômico-financeira propostos por Pereira et al. (2006) foram aplicados a partir da média das variáveis envolvidas, 
segundo o porte municipal. Para o cálculo dos indicadores na série histórica, foram observadas as seguintes características:

Despesa potencial em saúde per capita $(D P S P C)=(0,15 X R P P C)+(0,15 X$ TCPC) + TSUSPC.

Onde: RPPC: Receita própria per capita.

TCPC: Transferências constitucionais per capita.

TSUSPC: Transferências do SUS per capita.

Margem de expansão do gasto per capita $(M E G P C)=D P S P C-D A P C$

Onde: DPSPC: Despesa potencial em saúde per capita

DAPC: Despesa atual em saúde per capita.

Observe-se que o multiplicador 0,15 atende às aplicações mínimas de recursos municipais determinadas pela EC 29, como índice pleno a partir de 2004. As variáveis consideradas tiveram os per capitas dimensionados a partir da informação do Siops e foram extraídas médias por portes, levando em consideração a existência e eliminação de outliers dentro de cada porte populacional e exclusão dos municípios sem informação.

\section{Dimensão das despesas municipais com saúde}

Foram analisadas as relações entre as transferências do SUS e o gasto total com finalidade de entender o grau de dependência municipal das transferências federais e estaduais específicas da saúde. Essas transferências destinam-se ao financiamento dos programas do SUS, inclusive a AB.

\section{Dimensão da alocação municipal e das transferências federais para e evolução da cobertura das linhas de atuação estruturantes da $\mathrm{AB}$}

Foi avaliada a tendência de alocação de recursos em $A B$, por meio da aplicação na Subfunção Atenção Básica. Para esse fim, foram investigados o peso do gasto desta subfunção no gasto total com saúde e a evolução das transferências federais para o custeio das linhas de atuação ESF e ACS. 
Resultados

Dimensão das Receitas Disponíveis (RD)

Gráfico 1. Participação dos impostos de competência municipal na formação da receita própria

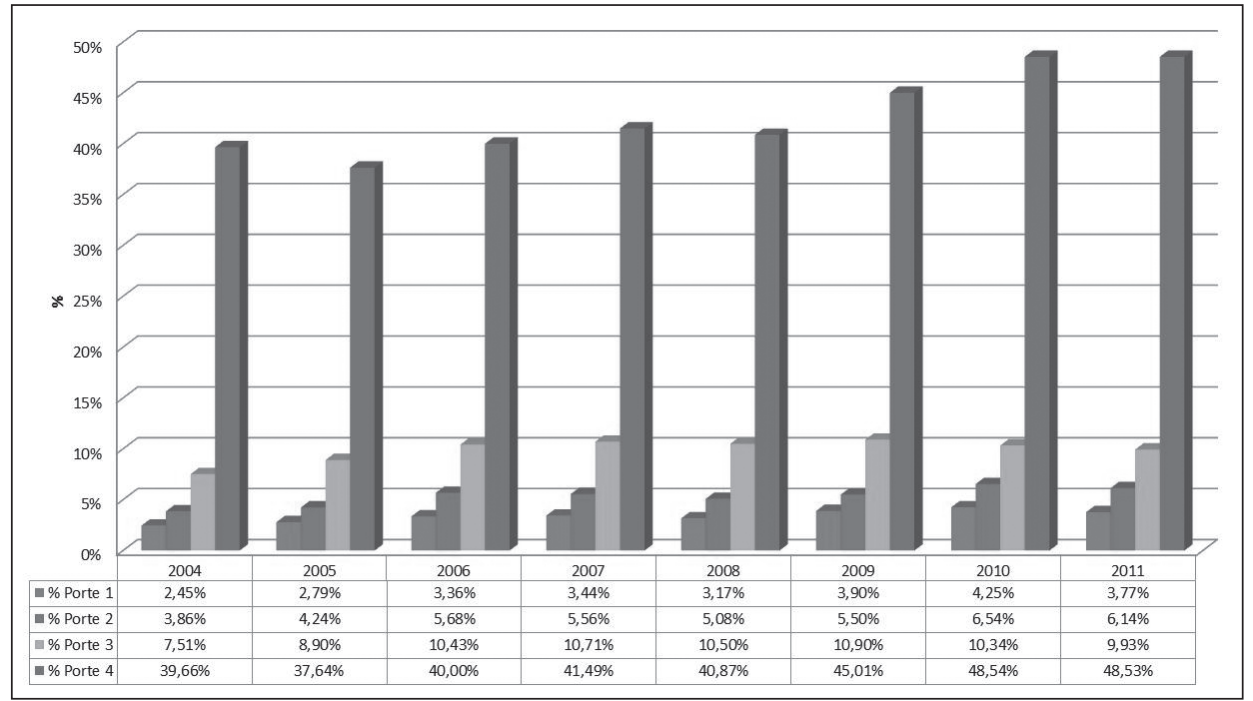

Fonte: MS/SIOPS

O gráfico 1 facilita a visualização da participação dos impostos na RD do conjunto de municípios de Minas Gerais selecionados para atuação do BSM, estratificados segundo o porte municipal. Observa-se que o peso dos impostos na formação da RD eleva-se proporcionalmente ao porte municipal, sendo bem mais destacado nos municípios com mais de 100 mil habitantes. Essa constatação indica que nos municípios menos populosos há maior dependência das transferências constitucionais e legais para a cobertura das políticas públicas em geral e das transferências do SUS para a política de saúde em particular. 
Tabela 1. Evolução da arrecadação de impostos de competência municipal (em \%)

\begin{tabular}{|l|c|c|c|c|c|c|c|}
\hline \multirow{2}{*}{$\begin{array}{l}\text { Porte } \\
\text { Municipal }\end{array}$} & \multicolumn{7}{|c|}{ Anos } \\
\cline { 2 - 8 } & $2005 / 2004$ & $2006 / 2005$ & $2007 / 2006$ & $2008 / 2007$ & $2009 / 2008$ & $2010 / 2009$ & $2011 / 2010$ \\
\hline 1 & 29,34 & 29,97 & 15,00 & $-8,26$ & 16,79 & 22,40 & 7,46 \\
\hline 2 & 23,76 & 40,61 & 8,21 & $-6,02$ & 3,62 & 33,96 & 16,06 \\
\hline 3 & 27,37 & 22,20 & 14,60 & $-2,33$ & 0,94 & 9,73 & 19,04 \\
\hline 4 & 0,30 & 9,66 & 11,62 & $-4,91$ & 6,51 & 29,64 & 19,09 \\
\hline
\end{tabular}

Fonte: Siops/SE/MS.

$\mathrm{Na}$ evolução no decorrer do período, percebem-se oscilações nessa relação em todos os portes populacionais apresentados na tabela 1, havendo maior intensidade nos dois estratos de municípios de porte mais reduzido e declinando segundo a elevação do porte municipal. Chama a atenção o recuo do ano de 2008 em relação a 2007, denotando queda mais significativa da arrecadação dos impostos municipais (5,38\% em média em todos os portes) que dos repasses sob a forma de transferências constitucionais e legais $(1,41 \%$ em média em todos os portes), como pode ser visto nas tabelas 2 e 3. Essa situação evidencia os reflexos da crise financeira internacional sobre as perspectivas de crescimento da economia brasileira naquele ano.

Tabela 2. Arrecadação per capita de impostos de competência municipal (em R\$1)

\begin{tabular}{|l|c|c|c|c|c|c|c|c|}
\hline \multirow{2}{*}{$\begin{array}{l}\text { Porte } \\
\text { Municipal }\end{array}$} & \multicolumn{7}{|c|}{ Anos } \\
\cline { 2 - 9 } & 2004 & 2005 & 2006 & 2007 & 2008 & 2009 & 2010 & 2011 \\
\hline 1 & 18,80 & 24,31 & 31,60 & 36,34 & 33,33 & 38,93 & 47,65 & 51,20 \\
\hline 2 & 17,36 & 21,49 & 30,21 & 32,69 & 30,72 & 31,83 & 42,65 & 49,49 \\
\hline 3 & 22,65 & 28,85 & 35,25 & 40,40 & 39,46 & 39,83 & 43,70 & 52,02 \\
\hline 4 & 178,96 & 179,50 & 196,84 & 219,72 & 208,94 & 222,53 & 288,50 & 343,57 \\
\hline
\end{tabular}

Fonte: Siops/SE/MS. 
Tabela 3. Valores per capita das transferências constitucionais e legais aos municípios

(em R\$ 1)

\begin{tabular}{|l|c|c|c|c|c|c|c|c|}
\hline \multirow{2}{*}{$\begin{array}{l}\text { Porte } \\
\text { Municipal }\end{array}$} & \multicolumn{7}{|c|}{ Anos } \\
\cline { 2 - 9 } & 2004 & 2005 & 2006 & 2007 & 2008 & 2009 & 2010 & 2011 \\
\hline 1 & 765,90 & 872,56 & 941,13 & $1.055,75$ & $1.051,63$ & 998,07 & $1.121,60$ & $1.358,83$ \\
\hline 2 & 450,22 & 507,05 & 531,92 & 587,91 & 604,31 & 579,11 & 652,46 & 805,84 \\
\hline 3 & 301,43 & 324,23 & 338,05 & 377,22 & 375,74 & 365,46 & 422,81 & 523,72 \\
\hline 4 & 451,23 & 476,85 & 492,09 & 529,55 & 511,21 & 494,44 & 594,32 & 707,88 \\
\hline
\end{tabular}

Fonte: Siops/SE/MS.

Ainda nas tabelas 2 e 3, verifica-se a superioridade do valor per capita das transferências recebidas sobre a arrecadação de impostos municipais, em todos os portes populacionais, evidenciando que a base vinculável para aplicação da EC 29 tem, em sua composição, uma sustentação mais robusta nas transferências constitucionais e legais, principalmente nos municípios de menor porte.

Tabela 4. Percentual médio de aplicação de aplicação de Receitas Disponíveis no gasto com ações e serviços públicos de saúde (em \%)

\begin{tabular}{|l|c|c|c|c|c|c|c|c|}
\hline \multirow{2}{*}{$\begin{array}{l}\text { Porte } \\
\text { Municipal }\end{array}$} & \multicolumn{7}{|c|}{ Anos } \\
\cline { 2 - 9 } & $\mathbf{2 0 0 4}$ & $\mathbf{2 0 0 5}$ & $\mathbf{2 0 0 6}$ & $\mathbf{2 0 0 7}$ & $\mathbf{2 0 0 8}$ & $\mathbf{2 0 0 9}$ & $\mathbf{2 0 1 0}$ & $\mathbf{2 0 1 1}$ \\
\hline 1 & 16,89 & 18,26 & 19,27 & 18,93 & 19,73 & 19,09 & 19,12 & 18,91 \\
\hline 2 & 16,01 & 18,50 & 20,21 & 19,58 & 19,97 & 19,93 & 19,95 & 19,50 \\
\hline 3 & 14,59 & 19,52 & 20,99 & 27,25 & 23,35 & 20,77 & 21,00 & 18,45 \\
\hline 4 & 20,67 & 21,61 & 23,92 & 23,31 & 23,37 & 23,63 & 23,17 & 23,38 \\
\hline
\end{tabular}

Fonte: Siops/SE/MS.

A Constituição Federal em vigor, pelo disposto na EC 29, determina o emprego de recursos municipais em ações e serviços públicos de saúde (ASPS) de, no mínimo, $15 \%$ das RD, com ajustes de nivelamento dos municípios até 2003. A partir de 2004, a aplicação dos $15 \%$ deveria ser plena. O indicador apresentado na tabela 4 aponta essa relação e demonstra a disposição dos governos 
1242 municipais em incrementar, com RD, o gasto público em saúde, com aplicações acima do percentual estipulado. A análise da situação dos municípios mineiros com atuação do BSM mostra que, em média, a maioria deles, de todos os portes populacionais, satisfez essa determinação. Para os municípios com mais de 50 mil habitantes, os percentuais do gasto apresentados afastam-se positivamente, com mais intensidade, do mínimo determinado pela EC 29. O crescimento da aplicação de RD, no entanto, foi apenas moderado de 2004 a 2011. Evoluiu, de acordo com os portes municipais, de 2,2 a 3,9 pontos percentuais. Apenas no Porte 3, verificou-se condição insatisfatória em 2004.

A tabela 5 aponta a evolução do cumprimento dessa determinação nos anos extremos da série e o percentual de variação verificado em cada porte municipal.

Tabela 5. Coeficiente de variação $(\mathrm{CV})$ do percentual médio de aplicação de aplicação de Receitas Disponíveis no gasto com açōes e serviços públicos de saúde em 2004 e 2011, por porte municipal

\begin{tabular}{l|c|c|c|c|c|c}
\hline \multirow{2}{*}{$\begin{array}{l}\text { Porte } \\
\text { Municipal }\end{array}$} & \multicolumn{5}{|c}{2004} & \multicolumn{2}{c}{ Anos } \\
\cline { 2 - 7 } & $\begin{array}{c}\text { Média } \\
(\%)\end{array}$ & DDP & CV (\%) & $\begin{array}{c}\text { Média } \\
(\%)\end{array}$ & DP & CV (\%) \\
\hline 1 & 16,89 & 4,66 & 28,00 & 18,91 & 2,86 & 15,00 \\
\hline 2 & 16,01 & 5,20 & 32,00 & 19,50 & 3,32 & 17,00 \\
\hline 3 & 15,59 & 2,49 & 17,00 & 18,45 & 2,99 & 16,00 \\
\hline 4 & 20,67 & 4,15 & 20,00 & 23,38 & 3,21 & 14,00 \\
\hline
\end{tabular}

Fonte: Elaboração própria a partir de dados do Siops.

Nota: DP - Desvio Padrão.

A evolução verificada entre os dois anos demonstra a disposição dos governos municipais em incrementar, com $\mathrm{RD}$, o gasto público em saúde, por meio das aplicaçôes acima do percentual estipulado. A análise dos dados de painel dos municípios mineiros com atuação do BSM mostra que, em 2011, em todos os estratos municipais, constata-se essa ocorrência. O CV indica notável homogeneidade de aplicações em torno da média em 2011. A análise 
conjunta do CV com a média per capita de aplicação, acima dos $15 \%$, revela o comprometimento municipal com o desenvolvimento do SUS.

Confirmou-se, para a amostra, o alinhamento apontado por Portela e Ribeiro (2011), para a Região Sudeste, nos municípios com mais de 100 mil habitantes, referente à intensificação da adequação ao cumprimento da EC 29. Os maiores percentuais de aplicação correspondem a maiores responsabilidades sanitárias assumidas pelos entes municipais. Os autores alertam que essa situação reflete a progressão da importância do orçamento da setorial nas despesas públicas locais.

Os resultados dos indicadores de sustentabilidade da disponibilidade de recursos propostos por Pereira et al. (2006) são apresentados nas tabelas 6 e 7. Segundo os autores, o indicador "Despesa potencial em saúde per capita (DPSPC)" permite inferências sobre a situação das disponibilidades financeiras municipais potencialmente aplicáveis em ações de saúde. A interpretação de Pereira et al. das variáveis que influenciam a dimensão da DPSPC destaca as transferências do SUS, predominantemente as federais. Essas transferências seguem uma sistematização de cálculo própria com forte influência do histórico da capacidade da oferta.

Tabela 6. Despesa potencial em saúde per capita - DPSPC (em R\$1)

\begin{tabular}{l|c|c|c|c|c|c|c|c}
\hline \multirow{2}{*}{$\begin{array}{l}\text { Porte } \\
\text { Municipal }\end{array}$} & 2004 & 2005 & 2006 & 2007 & 2008 & 2009 & 2010 & 2011 \\
\cline { 2 - 9 } & 182,30 & 212,01 & 259,52 & 272,06 & 265,05 & 267,52 & 319,48 & 372,62 \\
\hline 1 & 134,72 & 153,88 & 175,41 & 192,49 & 193,84 & 206,34 & 243,21 & 270,38 \\
\hline 3 & 138,75 & 146,06 & 153,82 & 170,99 & 169,97 & 195,50 & 242,00 & 246,11 \\
\hline 4 & 278,10 & 273,71 & 295,09 & 307,17 & 296,80 & 328,45 & 400,57 & 453,19 \\
\hline
\end{tabular}

Fonte: Siops/SE/MS.

A tabela 6 mostra os municípios com mais de 100 mil habitantes em situação favorável, com um per capita médio de DPSPC bem mais elevado que os demais. Levando em consideração a participação das transferências do SUS na fórmula de cálculo, esses valores podem ser influenciados pelo papel desses municípios como polo de oferta de serviços de média e alta complexidade computada nessas 
transferências. Nota-se que o resultado desse indicador é coerente com o apurado para os percentuais de RD aplicados em saúde (tabela 5).

A evolução da DPSPC ao longo da série evidencia que nos municípios com até 10 mil habitantes houve maior intensificação de recursos potencialmente disponíveis. Os municípios com mais de 100 mil habitantes apresentaram evolução mais branda.

Tabela 7. Coeficiente de variação $(\mathrm{CV})$ da despesa potencial em saúde per capita (DPSPC) em 2004 e 2011, por porte municipal

\begin{tabular}{l|c|c|c|c|c|c}
\hline \multirow{2}{*}{$\begin{array}{l}\text { Porte } \\
\text { Municipal }\end{array}$} & \multicolumn{5}{|c}{2004} & \multicolumn{2}{c}{ Anos } \\
\cline { 2 - 7 } & Média (R\$) & DP & CV (\%) & $\begin{array}{c}\text { Média } \\
(\mathrm{R} \$)\end{array}$ & DP & CV (\%) \\
\cline { 2 - 7 } & 182,30 & 68,71 & 31,19 & 372,62 & 105,10 & 27,52 \\
\hline 2 & 134,72 & 41,95 & 25,61 & 270,38 & 59,90 & 21,34 \\
\hline 3 & 138,75 & 22,64 & 15,50 & 246,11 & 39,25 & 15,56 \\
\hline 4 & 278,10 & 89,26 & 29,59 & 453,19 & 183,15 & 37,43 \\
\hline
\end{tabular}

Elaboração própria a partir de dados do Siops.

Nota: DP - Desvio Padrão.

A análise do CV nos anos extremos da série demonstra intensificação da homogeneidade municipal em relação à propensão de aplicar recursos em ações de saúde.

O indicador "margem de expansão do gasto per capita (MEGPC)", mostrado na tabela 8 , abate do valor apurado da DPSPC o gasto atual em ações de saúde para efeito da EC 29. De acordo com Pereira et al. (2006), esse indicador permite dois tipos de interpretação: 1) os municípios mais bem posicionados são os que têm menor margem de expansão do gasto em saúde, ou seja, já gastam com saúde além de sua despesa potencial - são aqueles com margem de expansão com menores resultados ou com resultados negativos; 2) os municípios que apresentam maior margem de expansão do gasto com saúde são aqueles que poderão ter mais facilidade de incorporar novas despesas à sua estrutura de gastos porque gastam aquém do seu potencial. 
Tabela 8. Margem de expansão do gasto per capita - MEGPC (em R \$ 1)

\begin{tabular}{l|c|c|c|c|c|c|c|c}
\hline \multirow{2}{*}{$\begin{array}{l}\text { Porte } \\
\text { Municipal }\end{array}$} & \multicolumn{7}{|c}{ Anos } \\
\cline { 2 - 9 } & 2004 & 2005 & 2006 & 2007 & 2008 & 2009 & 2010 & 2011 \\
\hline 1 & - & $-35,58$ & $-44,40$ & $-50,69$ & $-58,19$ & $-50,37$ & $-63,78$ & $-69,37$ \\
\hline 2 & - & $-29,09$ & $-30,22$ & $-29,01$ & $-41,88$ & $-39,00$ & $-54,17$ & $-57,35$ \\
\hline 3 & - & $-65,46$ & $-31,62$ & $-58,23$ & $-51,00$ & $-47,67$ & $-48,70$ & $-65,65$ \\
\hline 4 & - & $-110,27$ & $-67,04$ & $-75,60$ & $-70,67$ & $-68,11$ & $-80,49$ & $-113,50$ \\
\hline
\end{tabular}

Fonte: Siops/SE/MS.

Nota: Dados de despesas com ASPS indisponíveis para 2004 e valores negativos nos demais.

Pela primeira interpretação, os municípios com mais de 100 mil habitantes encontram-se mais bem posicionados em termos de montantes per capitas empregados em saúde, indicação de que têm maior comprometimento setorial de valores por habitante. De acordo com a segunda leitura, esses municípios apresentam menor possibilidade de expansão de gasto.

Os municípios com até 10 mil habitantes apresentam a segunda posição em termos de per capita empregado, segundo a primeira leitura do indicador. Essas posições reforçam os resultados per capita das DPSPC. Pela segunda interpretação, esses municípios também estariam na segunda posição no ranking de menor possibilidade de expansão do gasto per capita.

De acordo com a segunda interpretação, o estrato municipal com maior possiblidade de incorporar novas responsabilidades, em termos de expansão de atuação, seria aquele com mais de 10 mil até 50 mil habitantes.

Tabela 9. Coeficiente de variação (CV) da Margem de expansão do gasto per capita (MEGPC) em 2005 e 2011, por porte municipal

\begin{tabular}{l|c|c|c|c|c|c}
\hline \multirow{2}{*}{$\begin{array}{l}\text { Morte } \\
\text { Municipal }\end{array}$} & \multicolumn{5}{|c}{ Anos } \\
\cline { 2 - 7 } & Média (R\$) & DP & CV (\%) & Média (R\$) & DP & CV (\%) \\
\hline 1 & $-35,27$ & 50,73 & 143,83 & $-68,73$ & 52,58 & 76,50 \\
\hline 2 & $-29,09$ & 29,87 & 102,68 & $-57,35$ & 35,13 & 61,26 \\
\hline 3 & $-65,46$ & 84,09 & 128,46 & $-65,65$ & 17,44 & 26,57 \\
\hline 4 & $-110,27$ & 36,67 & 33,25 & $-113,50$ & 82,28 & 72,49 \\
\hline
\end{tabular}

Elaboração própria a partir de dados do Siops.

Nota: DP - Desvio Padrão 
A tabela 9 mostra que, pela primeira interpretação, os municípios com até 50 mil habitantes elevaram notavelmente seu comprometimento financeiro com ações de saúde entre 2005 e 2011. Além da intensificação da aplicação, o CV, obtido para 2011, demonstra aprimoramento na homogeneidade entre esses municípios com relação à mobilização de recursos financeiros para o setor. Pela segunda leitura, os municípios com até 50 mil habitantes apresentam maior margem de expansão e, portanto, estão mais propensos a expandirem sua atuação em saúde.

Entretanto, para Portela \& Ribeiro (2011), a leitura conjunta das duas interpretações sugere que os municípios com maior gasto per capita têm aparentemente mais alto grau de institucionalização de programas de saúde, indicando mais maturidade do gasto, o que favorece maior sustentabilidade econômico-financeira para a ampliação de metas das linhas de atuação já em operação.

A situação dos municípios de Minas Gerais com relação ao indicador de expansão de despesas, a MEGPC, é a demonstrada na tabela 10, tendo como referência o per capita médio da MEGPC.

Tabela 10. Situação dos municípios de Minas Gerais com relação à MEGPC

\begin{tabular}{l|c|c}
\hline \multirow{2}{*}{$\begin{array}{l}\text { Porte } \\
\text { Municipal }\end{array}$} & \multicolumn{2}{|c}{ No. De Municípios } \\
\cline { 2 - 3 } & Abaixo da média & Acima da média \\
\hline 1 & 46 & 36 \\
\hline 2 & 37 & 1 \\
\hline 3 & 2 & 3 \\
\hline 4
\end{tabular}

Elaboração própria. 
Gráfico 2. Participação das transferências do SUS nas despesas totais com saúde

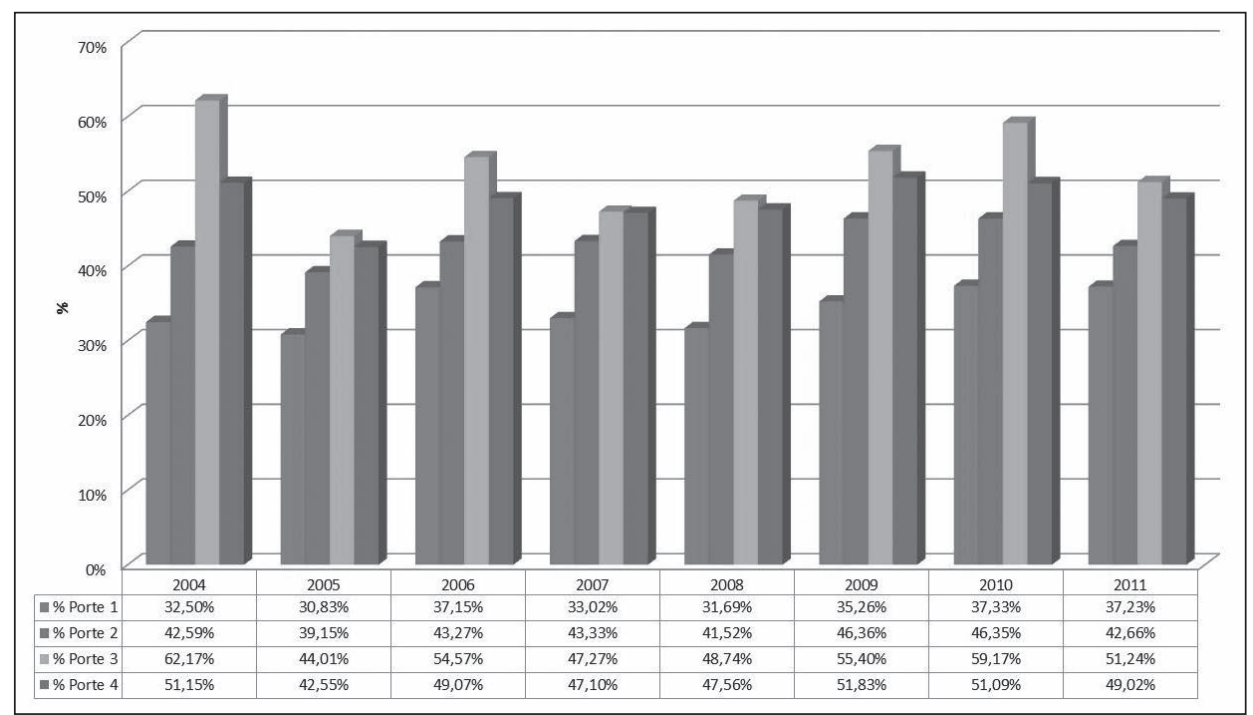

Fonte: MS/SIOPS

Para examinar a despesa municipal com saúde, levou-se em consideração o pressuposto de que municípios com menor arrecadação de impostos teriam maior dependência financeira das transferências em geral, e das do SUS em particular. Porém, o gráfico 2 mostra que os municípios menores (Portes 1 e 2) utilizam as transferências do SUS para cobertura de suas despesas totais em níveis abaixo da média do conjunto de municípios. Essa constatação contradiz o pressuposto ao comparar esse resultado com o observado no gráfico 1, em que esses dois estratos municipais têm níveis de impostos mais restritos na formação das RD. 

para e evolução da cobertura das linhas de atuação estruturantes da atenção básica

\section{Gráfico 3. Relação \% entre Atenção Básica e Gasto com Saúde Total}

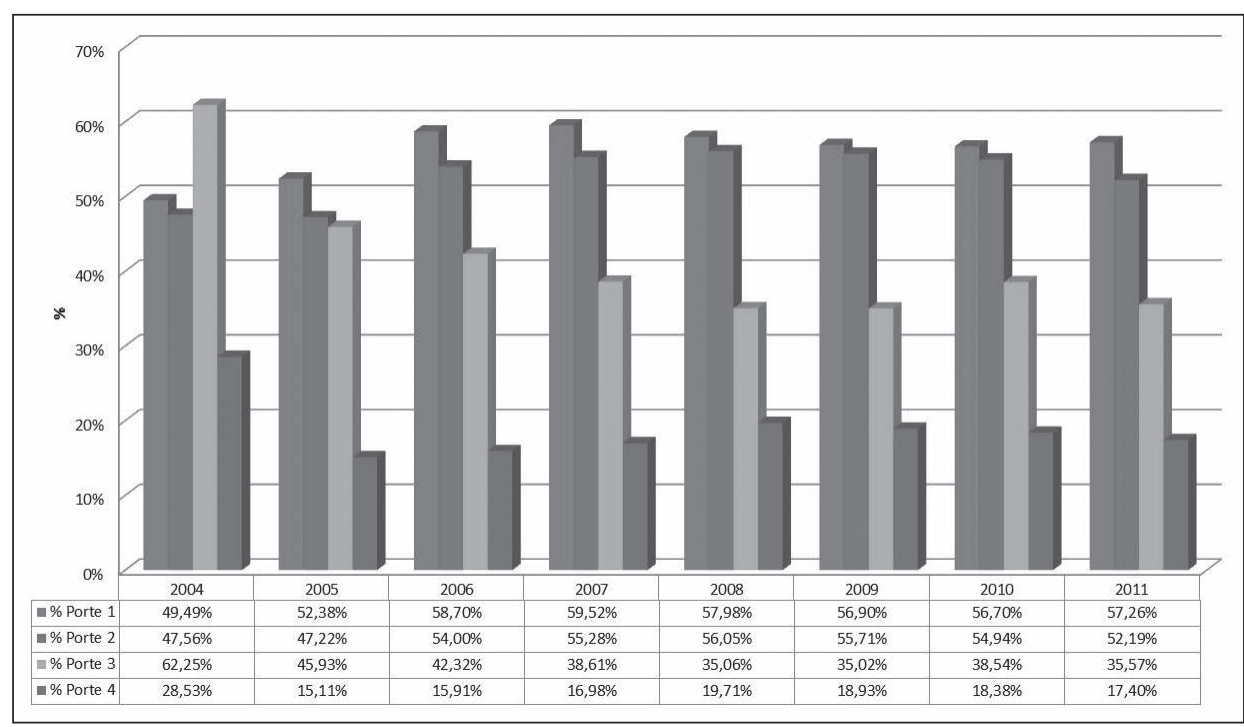

Fonte: MS/SIOPS

O gráfico 3 permite visualizar que, em relação ao gasto total com saúde, a proporção empregada na subfunção "Atenção Básica" decresce com o porte municipal, sendo mais elevada nos municípios com até 10 mil habitantes, com média de 56,12\% no período. Nos municípios com populaçōes entre 10 mil e 50 mil habitantes o movimento é semelhante, porém com proporções médias em torno de 52,87\%. Entre os de 50 mil e 100 mil, a média foi de 41,86\%. Nesses municípios, ao longo da série, observa-se ascensão da alocação em $\mathrm{AB}$ com tendência de estabilização a partir de 2006.

Nos dois estratos de maior porte, o movimento foi inverso desde o início do período. Nos municípios com mais de 100 mil habitantes, o gasto com a $\mathrm{AB}$ corresponde a uma média de $18,87 \%$ do gasto público com saúde no período, o que aparenta a preponderância de gastos com a atenção especializada, de média e alta complexidade, já que as demais linhas de atuação têm as correspondentes subfunções com despesas abaixo da $\mathrm{AB}$. 
Tabela 11. Valor per capita das transferências do Bloco da Atenção Básica seccionada em Equipes de Saúde da Família (em R \$1)

\begin{tabular}{|l|c|c|c|c|c|c|c|c|}
\hline \multirow{2}{*}{$\begin{array}{l}\text { Porte } \\
\text { Municipal }\end{array}$} & \multicolumn{7}{|c|}{ Anos } \\
\cline { 2 - 9 } & $\mathbf{2 0 0 4}$ & $\mathbf{2 0 0 5}$ & $\mathbf{2 0 0 6}$ & $\mathbf{2 0 0 7}$ & $\mathbf{2 0 0 8}$ & $\mathbf{2 0 0 9}$ & $\mathbf{2 0 1 0}$ & $\mathbf{2 0 1 1}$ \\
\hline 1 & 25,16 & 29,67 & 34,84 & 37,12 & 38,49 & 42,63 & 44,54 & 45,72 \\
\hline 2 & 20,11 & 23,17 & 29,34 & 31,03 & 39,65 & 36,25 & 37,30 & 37,08 \\
\hline 3 & 14,99 & 13,63 & 16,69 & 16,29 & 13,83 & 16,78 & 16,68 & 15,73 \\
\hline 4 & 10,91 & 9,82 & 10,07 & 10,73 & 11,02 & 11,91 & 11,38 & 12,00 \\
\hline
\end{tabular}

Fonte: Siops/SE/MS.

Tabela 12. Cobertura populacional por Equipes de Saúde da Família (em \%)

\begin{tabular}{|l|c|c|c|c|c|c|c|c|}
\hline \multirow{2}{*}{$\begin{array}{l}\text { Porte } \\
\text { Municipal }\end{array}$} & \multicolumn{7}{|c|}{ Anos } \\
\cline { 2 - 9 } & 2004 & 2005 & 2006 & 2007 & $\mathbf{2 0 0 8}$ & $\mathbf{2 0 0 9}$ & $\mathbf{2 0 1 0}$ & $\mathbf{2 0 1 1}$ \\
\hline 1 & 76,79 & 80,91 & 87,33 & 90,2 & 91,55 & 94,37 & 95,33 & 98,06 \\
\hline 2 & 65,33 & 70,95 & 80,42 & 83,37 & 88,39 & 88,49 & 93,56 & 95,77 \\
\hline 3 & 60,79 & 58,30 & 76,55 & 62,89 & 58,67 & 66,28 & 70,09 & 65,62 \\
\hline 4 & 41,24 & 40,46 & 43,70 & 45,46 & 48,09 & 47,12 & 46,33 & 51,01 \\
\hline
\end{tabular}

Fonte: Siops/SE/MS.

Com relação às transferências federais do Bloco da Atenção Básica, seccionadas na manutenção das equipes de saúde da família e dos agentes comunitários de saúde, pode-se verificar a tendência dessas transferências e as respectivas coberturas.

As tabelas 12 e 13 evidenciam que nos dois estratos de menor porte, enquanto o valor per capita transferido elevou-se em mais de $80 \%$, a cobertura populacional cresceu, em média, 27\% nos municípios com até 10 mil habitantes e $47 \%$ nos de até 50 mil habitantes. Nos municípios dos estratos mais elevados, enquanto as transferências per capita cresceram 5\% e 10\%, as coberturas populacionais elevaram-se em $8 \%$ e $24 \%$, respectivamente.

Essas relações sugerem que os municípios de menor porte utilizaram, com mais intensidade, as transferências do SUS para elevarem as coberturas populacionais por ESF, enquanto nos dois portes mais populosos, os municípios alcançaram maiores elevações de coberturas recorrendo a recursos das RD diante da evolução menos intensa das transferências do SUS. 
Tabela 13. Valor per capita das transferências do Bloco da Atenção Básica seccionada em Agentes Comunitários de Saúde (em R \$ 1)

\begin{tabular}{|l|c|c|c|c|c|c|c|c|}
\hline \multirow{2}{*}{$\begin{array}{l}\text { Porte } \\
\text { Municipal }\end{array}$} & \multicolumn{7}{|c|}{ Anos } \\
\cline { 2 - 9 } & $\mathbf{2 0 0 4}$ & $\mathbf{2 0 0 5}$ & $\mathbf{2 0 0 6}$ & $\mathbf{2 0 0 7}$ & $\mathbf{2 0 0 8}$ & $\mathbf{2 0 0 9}$ & $\mathbf{2 0 1 0}$ & $\mathbf{2 0 1 1}$ \\
\hline 1 & 9,12 & 9,87 & 12,29 & 16,38 & 19,53 & 20,34 & 22,44 & 23,83 \\
\hline 2 & 7,70 & 8,22 & 10,84 & 14,63 & 20,09 & 18,95 & 20,90 & 22,18 \\
\hline 3 & 6,99 & 7,65 & 9,43 & 13,21 & 13,80 & 14,76 & 15,73 & 17,26 \\
\hline 4 & 4,63 & 4,52 & 5,04 & 6,43 & 7,67 & 7,44 & 7,50 & 8,39 \\
\hline
\end{tabular}

Fonte: Siops/SE/MS.

Tabela 14. Cobertura populacional por Agentes Comunitário de Saúde (em \%)

\begin{tabular}{|l|c|c|c|c|c|c|c|c|}
\hline \multirow{2}{*}{$\begin{array}{l}\text { Porte } \\
\text { Municipal }\end{array}$} & \multicolumn{7}{|c|}{ Anos } \\
\cline { 2 - 9 } & $\mathbf{2 0 0 4}$ & $\mathbf{2 0 0 5}$ & $\mathbf{2 0 0 6}$ & $\mathbf{2 0 0 7}$ & $\mathbf{2 0 0 8}$ & $\mathbf{2 0 0 9}$ & $\mathbf{2 0 1 0}$ & $\mathbf{2 0 1 1}$ \\
\hline 1 & 94,28 & 94,71 & 98,42 & 97,21 & 97,15 & 98,51 & 97,84 & 99,52 \\
\hline 2 & 84,25 & 86,52 & 94,68 & 93,50 & 97,82 & 96,21 & 99,10 & 99,52 \\
\hline 3 & 78,22 & 77,69 & 92,50 & 91,18 & 86,86 & 84,55 & 87,06 & 90,54 \\
\hline 4 & 56,27 & 50,72 & 51,42 & 51,45 & 51,18 & 48,10 & 46,89 & 52,69 \\
\hline
\end{tabular}

Fonte: Siops/SE/MS.

A tabela 14 demonstra que a cobertura populacional pelos ACS, nos municípios com até 10 mil habitantes, conservou certa estabilidade no período e demonstrou ser bem mais intensa nos municípios com mais de 10 mil até 100 mil habitantes. Chama a atenção que nos municípios com mais de 100 mil habitantes houve certa inconstância e uma tendência declinante da cobertura a partir de 2004, com leve intensificação em 2011.

Em situação oposta verifica-se, na tabela 14, que houve expressivo crescimento do valor per capita médio empregado pelo conjunto de municípios para o custeio dessa atividade, principalmente nos municípios com até 100 mil habitantes. Nesse porte municipal observam-se movimentos opostos: enquanto a cobertura populacional decresceu 6,36\%, o valor per capita transferido para o custeio dos ACS elevou-se $81,4 \%$ no período. 


\section{Considerações finais}

As investigações realizadas com relação às $\mathrm{RD}$ mostram vantagem para os municípios com mais de 100 mil habitantes que contam com valores per capita de arrecadação de impostos bem superiores à média dos demais municípios. Os municípios com até 50 mil habitantes, e principalmente os com até 10 mil, são mais dependentes das transferências federais em geral para financiarem suas atividades, dada a debilidade de sua estrutura tributária. Esses municípios, de início, tiveram mais dificuldade de cumprimento da EC 29. Atualmente apresentam percentuais de aplicação satisfatórios.

Pode-se inferir que os municípios de menor porte, por terem participações das transferências do SUS nas despesas totais com ações de saúde menos elevadas, para manter os níveis de despesas potenciais, utilizam, com mais intensidade, as transferências constitucionais e legais. Essas características sugerem maior autonomia dos municípios com porte acima de 100 mil habitantes para implementarem a política de saúde de acordo com suas opções de gestão.

$\mathrm{O}$ emprego de RD disponíveis no financiamento das ASPS, em geral, apresenta registros acima do piso de $15 \%$ em quase todos os portes municipais no período examinado. Os percentuais médios mais elevados foram detectados nos municípios com mais de 100 mil habitantes, o que representa coerência com seus níveis das receitas de impostos.

Verificou-se a intensificação da utilização do per capita das transferências do SUS proporcionalmente à elevação do porte municipal. $\mathrm{O}$ indicativo gerado é que os municípios com até 50 mil habitantes tendo, de um lado, limitações de arrecadação de impostos e, de outro, as dos parâmetros das transferências do SUS, fortemente baseados no histórico da capacidade da oferta de serviços de média e alta complexidade, provavelmente recorram, com mais intensidade, às transferências constitucionais e legais para financiarem as ações de saúde. $\mathrm{O}$ peso mais elevado das transferências do SUS nos municípios com mais de 100 mil habitantes pode ser creditado à infraestrutura de serviços especializados, consolidada nesses municípios, ou a seu papel de polo regional de referenciamento.

Os níveis de alocação municipal na subfunção "Atenção Básica” não chegam a surpreender. Tradicionalmente, essa linha de atenção teve maior desenvolvimento nos municípios de menor porte. No entanto, o comportamento das transferências federais do bloco da Atenção Básica para a cobertura das ESF e custeio dos ACS, 
1252 ações estruturantes da atuação em $\mathrm{AB}$, revelam desproporcionalidade entre a evolução dos valores per capita transferidos pelo SUS e das coberturas alcançadas. Mesmo nos municípios com até 50 mil habitantes, onde se constatam coberturas mais extensas, há desproporção entre a evolução do per capita transferido pelo SUS e a cobertura obtida nos anos examinados. Essa situação cria espaço para o aprofundamento da questão por estudos específicos, observando as diferenças regionais e os vários arranjos que se constituem em cada estado ou município como formas alternativas de atuação.

Os indicadores de sustentabilidade propostos por Pereira et al. apontaram os municípios com mais de 100 mil habitantes com vantagem na dimensão da despesa potencial per capita, sinalizando que, para esses municípios, em média, há um potencial de aplicação de valores per capita mais intenso.

Em outra vertente, os municípios com menos de 50 mil habitantes apresentaram menores médias para as despesas potenciais justificadas pelas menores despesas com saúde por habitante. A margem de expansão do gasto per capita em saúde (MEGPC) apurada, indicador que mostra a disponibilidade financeira para expandir suas despesas em saúde, aponta menor disponibilidade de recursos para incrementos de despesas nos municípios com mais de 100 mil habitantes. Essa evidência representa menores possibilidades de incorporar a diversificação ou a ampliação de sua atuação setorial. Esse quadro é consequência das já elevadas despesas com saúde por habitante. Em situação semelhante, encontram-se os entes municipais com até 10 mil habitantes.

A situação dos municípios de Minas Gerais com relação ao per capita da MEGPC abaixo da média e acima desta é semelhante (tabela 10). Logo, as possibilidades de gastos suplementares em saúde são parecidas em todos os portes municipais, mas levando em consideração que aqueles com até 50 mil habitantes, 95,2\% da amostra, apresentam a desvantagem da maior dependência das transferências federais, suas condições tornam-se mais complexas.

$\mathrm{O}$ panorama traçado induz à conclusão de que, para os municípios com até 50 mil habitantes, a grande maioria daqueles com atuação no BSM, ampliarem suas metas ou linhas de atuação, nas propostas desse Plano, necessitarão ampliar sua capacidade tributária ou de recursos suplementares provenientes do incentivo financeiro específico do Governo Federal, dada a exígua margem de $\mathrm{RD}$ em que operam e os elevados níveis de cobertura em $\mathrm{AB}$ que já atingiram disponibilizando, principalmente, suas transferências constitucionais e legais. ${ }^{3}$ 


\section{Referências}

AFONSO, J.R.R.; ARAÚJO, E.A. A capacidade de gasto dos municípios brasileiros: arrecadação própria e receita disponível. Brasília: BNDES, 2011. Disponível em: http:// www.bndes.gov.br. Acesso em: 12 fev 2013.

CÂMARA, C.; BERMUDEZ, X.P. Notas sobre sustentabilidade, aids e poder público. In: RASIL. Ministério da Saúde. Sustentabilidade: aids e sociedade civil em debate. Secretaria de Vigilância em Saúde, Programa Nacional de DST e Aids. Brasília: Ministério da Sapude, 2004 (Série Parcerias e Mobilização Social, 5).

MENDES, A. Financiamento, gasto e gestão do Sistema Único de Saúde: a gestão descentralizada semiplena e plena do sistema municipal no Estado de São Paulo (19952001). Tese (Doutorado em Economia) - Instituto de Economia, Universidade Estadual de Campinas, Campinas, 2005. 422p.

MENDES, A. Financiamento e gasto do Sistema Único de Saúde na região metropolitana de São Paulo 2002-2008. 1a Ed. São Paulo: Observatório de Saúde da Região Metropolitana de São Paulo, 2010 (Caderno no 01, Série 1: Eixos Temáticos Financiamento e Gasto em Saúde).

PEREIRA, A.T.S. et al. A sustentabilidade econômico-financeira no Proesf em municípios do Amapá, Maranhão, Pará e Tocantins. Ciência e Saúde Coletiva, v. 11, n. 3, p. 607-620, 2006.

PORTELA, Z.P.; RIBEIRO, J.M. A sustentabilidade econômico-financeira da Estratégia Saúde da Família em municípios de grande porte. Ciência e Saúde Coletiva, v. 16, n. 3, p. 1719-1732, 2011.

ROCHA, F. et al. Mais recursos ou mais eficiência? Uma análise de oferta e de demanda por serviços de saúde no Brasil. Niterói: Anpec, 2012. Disponível em: http://www.anpec.org. br/encontro/2012/inscricao/files_I/i4-b1b4f9ecad97bb62cabcf280c07dc31a.pdf. Acesso em: 30 jan 2013.

\section{Legislação consultada}

BRASIL. Decreto no 7.492, de 2 de junho de 2011. Institui o Plano Brasil Sem Miséria. Diário Oficial [da] República Federativa do Brasil, Brasília, DF, Ano CXLVIII, n. 106, p. 6-7, jun. de 2011. Seção 1, pt. 1.

Decreto no 7.680, de 17 de fevereiro de 2012. Dispõe sobre a programação orçamentária e financeira, estabelece o cronograma mensal de desembolso do Poder Executivo para o exercício de 2012 e dá outras providências. Diário Oficial [da] República Federativa do Brasil, Brasília, DF, Ano CXLIX, n. 35-A, Edição Extra, p. 1-6, fev. de 2012, Seção 1 , pt. 1.

Emenda Constitucional no 29, de 13 de outubro de 2000. Altera a Constituição Federal de 1988 para assegurar recursos mínimos para o financiamento das ações e serviços públicos de saúde. Diário Oficial [da] República Federativa do Brasil, Brasília, DF, Ano CXXXVIII, n. 178 - E, p. 1-2, set. de 2000. Seção 1, pt.1. 

. Lei Complementar no 141, de 13 de janeiro de 2012. Regulamenta o art. 198 da Constituição Federal para dispor sobre valores mínimos aplicados anualmente pela União, Estados e Municípios. Diário Oficial [da] República Federativa do Brasil, Brasília, DF, Ano CXLIX, n. 11, p. 1-4, jan. de 2012. Seção 1, pt. 1.

. Lei $n^{\circ} 12.798$ de 4 de abril de 2013. Estima a receita e fixa a despesa da União para o exercício financeiro de 2013. Diário Oficial [da] República Federativa do Brasil, Brasília, DF, Ano CL, n. 65, p. 2-4, abr. de 2013. Seção 1, pt. 1.

Lei $\mathrm{n}^{\circ}$ 12.593, de 18 de janeiro de 2012. Institui o Plano Plurianual da União para o período de 2012 a 2015. Diário Oficial [da] República Federativa do Brasil, Brasília, DF, Ano CXLIX, n. 14, p. 1-2, jan. de 2012. Seção 1, pt. 1.

\section{Notas}

${ }^{1} \mathrm{O}$ boxplot é um gráfico que representa os quartis de uma distribuição, o intervalo interquartílico e os valores adjacentes superior e inferior. A distância interquartílica (DQ) corresponde ao intervalo entre o $1^{\circ}$. e o $3^{\circ}$. quartil. Os pontos que se encontram até 1,5 vezes da DQ são considerados externos e a mais de 3,0 vezes da DQ são denominados soltos. Em ambas as situaçôes, são considerados outliers, sendo que na primeira, dependendo do grau de dispersão, poderão ser conservados. No entanto, na segunda sua presença altera enormemente a média e a variabilidade dos dados e provocam conclusōes distorcidas.

${ }^{2} \mathrm{O}$ CV dimensiona o percentual de dispersão dos dados em relação à média. É calculado pela razão entre o desvio padrão e a média, multiplicada por 100.

${ }^{3}$ Os dois autores participaram igualmente da concepção do artigo, sendo que França foi responsável pela coleta e sistematização dos dados, resultados, discussóes e conclusôes. 


\section{Abstract}

Brazil without Misery Plan: the fragile revenue available in the municipalities of Minas Gerais state for the proposed funding of Primary Care This paper analyzes the economic and financial sustainability required to cover the goals of Primary Care in the municipalities of Minas Gerais state selected by the Federal Government to work in health under Brazil without Misery Plan (BSM). To develop the study, we investigated the databases of Siops/MS, Sage/MS and IBGE. Data were processed by statistical methods in order to deliver the results. To characterize the problem, population sizes were adopted. The indicators used were created by Pereira et al. (2006). Others indicators used were tested by Mendes (2010). The research was structured in three dimensions: resources originating from available revenues and SUS transfers; municipal health expenditure, with respective allocation; and federal transfers to Primary Care, with coverage of structural action lines. Results show that for municipalities with up to 50,000 inhabitants, $95.2 \%$ of total, to broaden their targets or lines of action proposed in the plan, they will need to enhance their capacity to tax or raise additional resources in other spheres, given the meager margin of available revenues in which they operate and the high levels of coverage in Primary Care already reached using mainly their constitutional and legal shipments.

> Key words: public policies; health policy; public management; health expenditures; Brasil sem Miséria plan. 